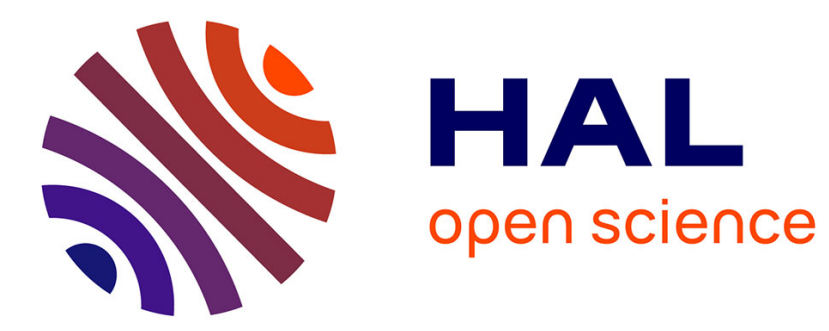

\title{
Vivre en centre maternel : le jeune enfant au cœur de la dynamique relationnelle entre sa mère et les professionnel-le-s
}

Claire Ganne

\section{- To cite this version: \\ Claire Ganne. Vivre en centre maternel : le jeune enfant au cœur de la dynamique relationnelle entre sa mère et les professionnel-le-s. Revue internationale de l'éducation familiale, 2017, 42 (2), pp.135-154. 10.3917/rief.042.0135 . hal-01960767}

\section{HAL Id: hal-01960767 \\ https://hal.parisnanterre.fr/hal-01960767}

Submitted on 19 Dec 2018

HAL is a multi-disciplinary open access archive for the deposit and dissemination of scientific research documents, whether they are published or not. The documents may come from teaching and research institutions in France or abroad, or from public or private research centers.
L'archive ouverte pluridisciplinaire HAL, est destinée au dépôt et à la diffusion de documents scientifiques de niveau recherche, publiés ou non, émanant des établissements d'enseignement et de recherche français ou étrangers, des laboratoires publics ou privés. 



\title{
Vivre en centre maternel : le jeune enfant au cour de la dynamique relationnelle entre sa mère et les professionnel-le-s
}

\author{
Claire Ganne ${ }^{1}$
}

En France, les établissements d'accueil mère-enfant, appelés également centres maternels, hébergent des femmes enceintes ou accompagnées d'enfant de moins de trois ans, dans le cadre de la protection de l'enfance. Ils ont pour mission d'apporter une aide éducative et psychologique aux femmes hébergées et de les soutenir dans leur relation avec leur enfant. Ce dernier est souvent accueilli en crèche au sein de l'établissement et est au centre des préoccupations des professionnel-le-s. Comment ces interventions sont-elles vécues du côté des mères? Cet article se base sur 49 entretiens biographiques réalisés auprès de femmes ayant séjourné en centre maternel quelques années. La restitution de leur expérience met en évidence le vécu du suivi éducatif, les configurations dans lesquelles il est perçu comme un soutien ou comme un contrôle, ainsi que la dynamique relationnelle paradoxale qui s'engage parfois.

Mots-clés : protection de l'enfance, travail social, centre maternel, soutien à la parentalité.

Living in a mother/child institution : the infant at the heart of the relationships between his mother and the professionals

French mother/child institutions, more commonly known as "centre maternel" (maternal centers), house pregnant women and women with their infants until their child is three years old. These "maternal centers" are part of child protection services. These institutions provide educational and psychological assistance to women, and support the mother-child relationship. The children are often

\footnotetext{
${ }^{1}$ Claire Ganne, CREF (EA 1589), Université Paris Nanterre, 200 avenue de l’Université, 92000 Nanterre, France.

Contact : ganne.c@u-paris10.fr
} 
welcomed in day-care centers inside the institution and are a core concern for the professionals. How do mothers experience these interventions? This paper is based upon 49 biographical interviews with women who were accommodated in maternal centers few years back. The restitution of their experience highlights the experience of the educational follow-up, the configurations in which it is perceived as a support and those where it is perceived as a control, and a paradoxical relationship often reveals itself.

Key-words : child welfare, social work, maternal center, parental support.

En France, les établissements d'accueil mère-enfant, plus communément appelés centres maternels, hébergent des femmes enceintes ou accompagnées d'enfant de moins de trois ans dans le cadre de la protection de l'enfance. Au 31 décembre 2012, on recensait 171 établissements d'accueil mèreenfants (Pliquet, 2015a), et 2300 mères isolées accompagnées de leurs enfants étaient hébergées dans ces structures (Pliquet, 2015b). Ces établissements relèvent de la protection de l'enfance, et accueillent des mères et des enfants présentant un cumul de fragilités. Après avoir décrit le contexte et les logiques d'intervention de ces établissements, nous nous centrerons sur le point de vue des femmes accueillies sur leurs relations avec les professionnel-le-s dans un tel contexte, sur la place de l'enfant au sein de ces dynamiques relationnelles et sur les interactions autour du moment de la sortie du centre maternel, qui cristallise les dynamiques relationnelles antérieures.

\section{L'accueil en centre maternel : contexte et logiques d'intervention}

\section{Un dispositif d'hébergement en direction de mères et de jeunes enfants}

Créés au cours du XIXe siècle pour prévenir les abandons et les infanticides en hébergeant les «filles mères » et leurs enfants, les centres maternels ont vu leurs missions et leurs logiques d'intervention évoluer au cours du XXe siècle, passant d'une visée morale et rééducative à une logique de soutien éducatif et psychologique en direction de mères isolées en difficulté (Boulbès, 2005). Les objectifs de survie sanitaire de l'enfant et de « redressement moral » de la mère ont laissé place à des missions plus éducatives. En effet, l'accroissement des connaissances sur le 
développement de l'enfant et les interactions précoces a amené l'idée de soutenir la relation mère-enfant au cours des premières années de vie. Toujours inscrits dans une logique préventive, ces établissements font partie des services concourant à la protection de l'enfance. Il en existe au minimum un par département. Leurs missions actuelles, fixées par une circulaire datant de 1981, sont très larges. Il s'agit de " rompre l'isolement des femmes enceintes et des jeunes mères en difficulté, en leur donnant la possibilité de s'exprimer, de s'informer et de nouer une relation (...) ; leur apporter l'aide matérielle dont elles ont besoin ; étant donné que ces personnes disposent d'un revenu au minimum égal, pour la quasi-totalité d'entre elles, à l'allocation de parent isolé, leur principal problème est celui de leur hébergement et de la garde de leur enfant; leur apporter une aide éducative ou psychologique et encourager leur réinsertion sociale, afin notamment de leur permettre de mieux se situer par rapport à la maternité, la sexualité, la vie en couple éventuellement, etc. ; le cas échéant, de former un projet de vie pour leur enfant; de former et réaliser un projet d'insertion socio-professionnelle » (Ministère de la Santé et de la Sécurité Sociale, 1981).

L'admission d'une femme enceinte ou accompagnée d'un enfant en centre maternel s'effectue avec son accord ${ }^{1}$, à la demande de différents services sociaux (de maternité, de secteur, de la protection de l'enfance...) et avec l'accord de l'Aide sociale à l'enfance, qui finance le séjour pour une durée de six mois. Cette prise en charge peut être renouvelée jusqu'à ce que l'enfant atteigne l'âge de trois ans. Pour cela, les travailleurs sociaux et les travailleuses sociales de la structure doivent transmettre un rapport d'évolution à l'Aide sociale à l'enfance, indiquant l'évolution de la situation de la famille et les grands axes du suivi éducatif réalisé.

Les femmes accueillies dans ces centres cumulent un grand nombre de fragilités : faible niveau scolaire, précarité socioéconomique, parcours de vie marqué par des problèmes de santé, des violences physiques ou sexuelles durant l'enfance, l'adolescence et à l'âge adulte (Corbillon et Duléry, 1997 ; Ganne et Bergonnier-Dupuy, 2011; Wendland, 2010). Si certains centres accueillent plus spécifiquement les mineures, celles-ci sont minoritaires parmi les femmes accueillies.

\footnotetext{
${ }^{1}$ Sauf dans le cas particulier de l'accueil de mères mineures faisant l'objet d'un placement par le juge des enfants.
} 


\section{Des logiques d'intervention centrées sur le lien mère-enfant}

Bien que l'admission s'effectue entre autres en raison d'une absence d'hébergement, l'action de ces établissements s'inscrit bien dans le cadre de la protection de l'enfance et non de l'hébergement d'insertion. Des recherches menées dans les années 1990 montrent la tension existante dans les logiques d'accompagnement de ces centres : les professionnel-le-s affirment en général l'importance du soutien à la relation mère-enfant; si l'insertion de la femme est également travaillée, l'articulation et la hiérarchisation de ces deux objectifs varient selon les établissements (Donati, Mollo-Bouvier, Norvez et Rollet-Echalier, 1999). Corbillon et Duléry (1997) constatent également l'importance de l'objectif de prévention des mauvais traitements et des négligences, présupposant des carences relationnelles du côté de la mère. Ils émettent l'hypothèse que cet objectif de prévention puisse interférer directement dans le processus de l'intervention, en entraînant des réactions d'hostilité de la part des mères, contradictoire avec l'objectif visé.

Ces questionnements font écho aux travaux des chercheur-e-s travaillant sur les interventions précoces (early interventions), qui prennent en compte le rôle des parents comme premiers donneurs de soin, les interactions entre enfants et parents et le soutien à la fonction parentale pour agir sur le développement de l'enfant (Korfmacher, 2002). Les différentes revues de littérature soulignent en général l'impact positif de ces interventions à domicile sur le développement des enfants et les relations parents-enfants, avec toutefois de meilleurs résultats auprès de certains types de publics et, au contraire, leur faible efficacité lorsque le cumul de facteurs de risque est trop important (Foll et Guedeney, 2014; Korfmacher, 2002). Elles soulignent également la nécessité de créer un fort lien d'alliance avec les familles concernées par ces programmes, ce qui est souvent rendu difficile par leur peur de l'intrusion des services sociaux (Dumaret, 2003).

De plus, Cardi (2008) constate que l'action éducative dans un centre maternel est également axée sur l'apprentissage de compétences domestiques et assigne ces femmes originaires de milieux populaires à un rôle féminin domestique traditionnel, en l'extrayant de ses relations sociales et conjugales. Elle souligne le caractère traditionnaliste du discours des professionnel-le-s de la protection de l'enfance concernant les femmes des classes populaires : quand la mère sort de son rôle traditionnel et de la sphère familiale, elle est considérée comme déviante. Tout engagement à l'extérieur du 
foyer est considéré comme allant à l'encontre de l'intérêt de l'enfant et de la société (Cardi, 2007).

On retrouve ce phénomène dans l'ensemble des interventions relevant de la protection de l'enfance et du soutien à la parentalité, majoritairement adressées aux mères issues des milieux populaires. Dans un contexte de responsabilisation massive des parents concernant les difficultés de leurs enfants (Neyrand, 2011), le développement de l'enfant peut devenir un enjeu entre professionnelle-s et familles. Ainsi, pour Wilpert (2013), plus les lieux d'accueil de la petite enfance se soucient de prévention voire de protection de l'enfance, "plus cette représentation d'une responsabilité maternelle exclusive, et confinée dans un espace familial clos, risque d'être écrasante pour les femmes réelles, qui, de plus, sont souvent les seules accessibles aux travailleurs sociaux, et par ce fait même risquent de porter tout le poids des difficultés familiales » (p. 76).

Ainsi, la situation des familles accueillies dans les centres maternels est révélatrice de plusieurs processus interdépendants. Leur situation familiale, économique et leur histoire personnelle favorisent la représentation spécifique de familles à haut risque et la centration des intervenants sur le «lien mère-enfant» ou les «compétences parentales », avec l'idée de prévenir des situations de difficultés éducatives ultérieures.

\section{Le cadre de la recherche}

Cet article, qui se centre plus spécifiquement sur l'analyse des relations entre les mères et les professionnel-le-s durant l'accueil en centre maternel, se base sur des données de plus grande ampleur recueillies dans le cadre d'une thèse de doctorat de Sciences de l'éducation, qui portait sur les processus de construction des parcours d'enfants après un accueil en centre maternel. L'objectif général de la recherche était de comprendre les processus de construction des parcours après un accueil en centre maternel, en utilisant notamment le cadre théorique de l'écologie du développement humain pour analyser les interactions autour de l'enfant.

\section{Une lecture écologique des processus}

Selon Bronfenbrenner (1996), le développement humain se produit grâce à des interactions prolongées de l'enfant avec les systèmes qui l'entourent. Au plus proche de l'enfant, se trouvent les différents microsystèmes dans lesquels l'enfant interagit directement : il s'agit d'un ensemble d'activités, de rôles sociaux et de relations 
interpersonnelles qu'expérimente l'enfant. Les enfants, même très jeunes, évoluent dans de nombreux microsystèmes : famille, crèche, autres lieux de garde, famille élargie, voisinage... Les enfants accueillis en centre maternel baignent dans un univers d'interactions multiples : ils ou elles habitent en général dans une chambre ou un studio avec leur mère et, dans le même bâtiment vivent également d'autres mères et d'autres enfants. Des professionnel-le-s, notamment des éducateurs et des éducatrices, sont présent-e-s sur des temps quotidiens. De plus, la plupart des centres proposent un accueil en crèche ou en halte-garderie.

Les interactions de l'enfant avec les personnes et les objets présents dans son environnement immédiat constituent les processus proximaux, qui soutiennent le développement de l'enfant. Or pour que ceux-ci soient efficaces, ils doivent survenir de manière constante et durable, sur une longue période de temps; cette stabilité est particulièrement dépendante des autres niveaux de l'écosystème. Les relations entre ces différents microsystèmes forment le mésosystème, susceptible d'influer sur la vie de l'enfant au sein des différents microsystèmes. L'exosystème rassemble les systèmes relationnels qui n'agissent pas directement sur l'enfant mais peuvent avoir des conséquences par le biais de leur action sur l'un des acteurs d'un microsystème. Enfin, le macrosystème est constitué par l'ensemble des valeurs, des normes et des lois qui structurent la société dans laquelle ces différents systèmes se développent.

Dans le cadre d'un accueil en centre maternel, le mésosystème, et notamment les relations entre les mères et les professionnel-le-s, peuvent être producteurs de stabilité ou d'instabilité. En effet, au niveau macrosystémique, les centres maternels sont inscrits dans le dispositif de protection de l'enfance. Cette inscription peut avoir une incidence sur les processus mésosystémiques, puisque d'autres recherches ont montré que les professionnel-le-s centraient beaucoup leur action et leur regard sur les pratiques éducatives des mères et l'évolution de l'enfant (Donati et al., 1999).

C'est pourquoi nous avons souhaité approfondir, dans cet article, la manière dont cette centration sur le lien mère-enfant était vécue par les mères, l'impact que cela avait eu sur leurs relations avec les professionnel-le-s, la place de l'enfant dans ces relations et les conséquences sur le contexte de développement de celui-ci. 


\section{Caractéristiques des parcours des femmes accueillies}

Afin de décrire et comprendre les processus de construction des parcours, une méthodologie catamnestique, consistant à retrouver le maximum de personnes ayant vécu un événement commun dans le passé (Corbillon, Assailly et Duyme, 1990), a été mise en place en partenariat avec deux départements français, afin de saisir les parcours environ huit ans après la sortie. Le recueil de données réalisé comprenait plusieurs étapes distinctes, dont un travail sur dossiers et des entretiens auprès de femmes anciennement hébergées. Le travail sur les dossiers a permis de décrire les caractéristiques d'environ 300 femmes hébergées en centre maternel au début des années 2000 (Ganne et Bergonnier-Dupuy, 2011). Trois grandes catégories de femmes accueillies ont été distinguées : les jeunes femmes accueillies avant l'âge de 21 ans, les migrantes adultes et les femmes adultes cumulant des difficultés psycho-sociales (handicap, addictions, problèmes psychiatriques...). Les jeunes femmes accueillies avant l'âge de 21 ans représentent la moitié des femmes hébergées dans les deux départements enquêtés. Pour une partie d'entre elles, elles vivaient avec leurs parents avant d'arriver au centre maternel, mais la grossesse a entraîné une situation de conflit ou de rupture familiale. Environ la moitié de ces jeunes femmes ont fait l'objet de mesures de protection de l'enfance en tant qu'enfant, soit dans le cadre d'un placement, soit dans le cadre d'une mesure de milieu ouvert. Parmi les femmes âgées de 21 ans et plus au moment de leur accueil, on peut distinguer deux groupes de femmes, diversement représentés selon les territoires : les migrantes adultes, arrivées en France depuis moins de deux ans, sont majoritaires dans le département d'Ile-de-France, alors que les femmes nées en France, suivies par des services sociaux en raison de difficultés sociales ou psychologiques, sont majoritaires dans le département de province enquêté. Elles ont très souvent été elles-mêmes placées durant leur enfance.

\section{Des entretiens auprès de 49 femmes}

En accord avec la Commission Nationale de l'Informatique et des Libertés et les services concernés, toutes les femmes dont les dossiers ont été étudiés et qui ont pu être localisées ont été sollicitées par courrier pour participer à un entretien de recherche. Des relances téléphoniques ont également été effectuées lorsque cela s'avérait possible. Dans la majorité des cas, les contacts avec les femmes ont été directement pris par la chercheuse, sur la base des adresses les plus récentes consignées dans les dossiers sociaux. Les contacts entre anciennes résidentes ont permis de recontacter certaines femmes. Les 
centres maternels des deux départements concernés ont également servi de relais lorsqu'ils avaient encore des contacts avec d'anciennes résidentes.

Les 49 femmes rencontrées en entretien ont été accueillies avec leurs enfants âgés de moins de trois ans dans une douzaine de centres maternels différents. Au moment de l'enquête, elles avaient quitté ces structures depuis sept à huit ans. La diversité des parcours observée dans la phase quantitative se retrouve chez les femmes enquêtées. Ainsi, 26 femmes sur 49 ont été accueillies avant l'âge de 21 ans, et parmi elles, 14 étaient suivies par l'Aide sociale à l'enfance dans le cadre d'un placement, d'une mesure de milieu ouvert ou d'un contrat jeune majeur avant leur accueil. Les 23 femmes accueillies après l'âge de 21 ans ont connu des parcours marqués par une migration récente pour huit d'entre elles et par des expériences antérieures avec les services sociaux en raison de leur situation : violences conjugales ( 8 femmes), handicap (4) et addictions (3) notamment.

Les 49 entretiens biographiques réalisés avaient pour objectif de comprendre le processus de construction des parcours ultérieurs et de saisir l'importance des dynamiques mésosystémiques autour de l'enfant. Ces entretiens ont permis de reconstituer les parcours des femmes et des enfants depuis la sortie et d'appréhender les perceptions des femmes sur leur séjour au centre maternel. Ces entretiens ont fait l'objet d'une analyse thématique de contenu. Nous nous centrons ici sur l'analyse des processus mésosystémiques et, plus précisément, sur la perception, par les femmes rencontrées, de leurs relations avec les professionnel-le-s des centres maternels durant leur accueil, alors que leur enfant était très jeune.

\section{Principaux résultats}

L'analyse des entretiens menés avec les femmes met en évidence des perceptions contrastées des relations qu'elles ont pu entretenir avec les professionnel-le-s, et notamment avec les personnes chargées d'assurer un suivi socio-éducatif. Les missions des centres maternels étant très larges, ce suivi concerne l'ensemble des dimensions de l'existence des femmes accueillies : prise en charge quotidienne du bébé, relation avec le père de l'enfant, suivi psychologique, projet professionnel, recherche de logement... Ce suivi prend en général la forme de rencontres fréquentes avec un-e référent-e social-e, le plus souvent une éducatrice, lors d'entretiens formels ou informels. Suivant les centres maternels, sa mise en œuvre varie : dans certains cas, il s'agira d'entretiens formels dans le cadre de rendez-vous réguliers imposés, alors que dans d'autres centres, les éducateurs et éducatrices 
s'appuieront davantage sur les échanges informels du quotidien, ce qui n'exclut pas des rendez-vous plus formels. De manière générale, plus cette parole est sollicitée sous une forme informelle, proche d'une vie sociale ou familiale «classique» (jeux de société le soir, café le matin...), mieux cette demande est acceptée par les résidentes.

Au-delà de la forme qu'il prend, la perception de ce suivi socioéducatif est particulièrement en lien avec le parcours des femmes accueillies : si les femmes les plus jeunes le vivent comme un soutien, voire une forme de suppléance familiale, les femmes adultes s'inscrivent dans d'autres logiques relationnelles. Elles manifestent souvent des réticences lorsque le suivi se focalise sur leurs compétences maternelles et l'évolution de l'enfant.

\section{Une fonction de suppléance pour les femmes les plus jeunes}

Une partie des femmes, et notamment les plus jeunes, estiment que les conseils des professionnel-le-s leur ont permis d'apprendre à s'occuper de leur bébé. Les conseils de puériculture et l'apprentissage de rythmes quotidiens sont évalués positivement. Plusieurs d'entre elles disent explicitement que c'est le centre maternel qui leur a appris à être maman, se suppléant ainsi au rôle de leur famille : "c'est pas mes parents qui m'ont appris à élever un enfant, à faire le bien pour un enfant, c'est le foyer, vu que j'étais toujours au foyer, donc tout ce que je fais automatiquement ça revient de là-bas parce que c'est làbas que j'ai appris à être une maman quoi ». Savoir donner à l'enfant des repères quotidiens, être capable de reconnaître les désagréments vécus par les bébés (coliques, percées dentaires...) sont mis en avant comme des compétences acquises au centre maternel. À ce titre, les apprentissages domestiques traditionnels réalisés au centre sont également valorisés par ces jeunes femmes : savoir cuisiner, recoudre un bouton, choisir des produits ménagers adaptés... Ces femmes, qui reconnaissent les apports éducatifs concernant les soins à apporter à un enfant, ont toutes été accueillies avant l'âge de vingt ans au centre maternel.

Ces jeunes femmes considèrent de plus que l'écoute des professionnel-le-s et les échanges réguliers avec eux ont été d'une grande aide : "Voilà c'est sûr que quand tu es épaulée, je vous dis le soutien des éducateurs, assistantes sociales, les dames de la $\mathrm{PMI}^{1} \ldots$ Pour le suivi de l'enfant c'est très important, beaucoup parler, parler, parler, voilà ». La demande de parole que leur adressent les professionnel-le-s est alors vécue comme une manière de prendre soin,

\footnotetext{
${ }^{1}$ Protection Maternelle et Infantile
} 
de se soucier d'elles et de leur enfant. "Ils m'ont beaucoup soutenue en fait, ils m'ont pas laissée à part, je veux dire c'était pas : tu as un studio, tu te débrouilles. Non ils m'ont bien soutenue moralement, tout ce qui était suivi avec l'enfant, tout ça, si ça allait, ils étaient toujours en demande si moi j'avais une demande ils étaient là à l'écoute... C'est une très très bonne structure je regrette pas. »

Dans ces situations, les femmes évoquent leurs relations avec les éducatrices en utilisant le registre des relations amicales et surtout familiales. Ainsi, l'une d'elles «s'est prise d'amitié» pour sa première éducatrice. Pour une autre, on peut parler aux éducateurs "comme à des amis». La référence euphémisée à la famille est largement présente, elle englobe en général à la fois les résidentes et les professionnel-le-s: "Je m'étais un peu attachée à eux je pense, c'était devenu comme une petite famille», "C'était plus un cocon familial que la structure éducative en fait», "Même au foyer ils disaient que c'était une petite famille», "Pour moi c'était une sorte de famille qui était autour de moi ». "Elle jouait avec nous, on rigolait ensemble et puis on parlait de tout de tout de tout, quand je vous dis de tout c'est de tout quoi, c'est vraiment comme si c'était notre grande sœur ou notre mère quoi... ». On peut ainsi considérer que les professionnel-le-s des centres maternels jouent, pour ces jeunes femmes ayant peu de soutien de leur propre famille, un rôle de suppléance familiale (Durning, 1995) au moment de l'arrivée de leur premier enfant.

La personnalisation des relations avec les professionnel-le-s tient ainsi une grande place dans le vécu du soutien. Ainsi, on constate à plusieurs reprises des difficultés en raison de changements de référente sociale. Parfois, ce changement permet la mise en place d'une nouvelle dynamique relationnelle, mais dans la plupart des récits, le départ d'une éducatrice avec qui le lien était important bloque complètement les échanges avec la nouvelle personne et induit une dynamique négative. Les changements multiples, dans certaines institutions où le turn-over des professionnel-le-s est important, fragilisent la dynamique relationnelle. Ainsi, l'une des femmes rencontrées aura été accompagnée par sept référent-e-s différent-e-s au cours de son séjour qui a duré deux ans.

\section{Une intervention éducative plus difficile à accepter pour les femmes adultes}

Par contre, la majorité des femmes accueillies après l'âge de 21 ans, tiennent à exprimer le fait qu'elles savaient s'y prendre avec un enfant. Il s'agit de femmes qui sont arrivées récemment en France, 
après un parcours migratoire, ou de femmes nées en France déjà suivies à de nombreuses reprises par des services sociaux (en raison d'un handicap, du placement d'un enfant aîné, de l'hébergement en structure d'insertion...).

Si les échanges portant sur les projets professionnels, les recherches de logement et les démarches administratives sont en général acceptés sans difficulté par ces femmes, ce n'est plus le cas lorsque le suivi touche à des dimensions plus personnelles (histoire familiale, relation avec le père de l'enfant...). La nécessité d'affirmer leurs compétences maternelles semble d'autant plus forte pour les femmes qui ont des enfants aînés non accueillis avec elles, qu'ils ou elles soient confié-e-s à la famille dans le pays d'origine pour les migrantes ou à l'Aide sociale à l'enfance pour les femmes déjà suivies par les services sociaux. Dès que des questionnements concernant le développement ou la prise en charge de l'enfant sont formulés par les professionnel-le-s, ils sont considérés comme inadaptés et ressentis de manière intrusive et disqualifiante. Des tensions peuvent facilement apparaître lorsque le personnel de la crèche souligne des points à surveiller dans le développement de l'enfant (notamment concernant le sommeil, l'alimentation) ou estime que le rythme proposé par la mère (par exemple pour l'apprentissage de la propreté) n'est pas adapté.

De plus, ces femmes ont parfois eu le sentiment que les professionnel-le-s de la crèche ou du centre maternel tentent de leur imposer des normes éducatives qui ne correspondent pas à leur système de référence, que ce soit en raison de leur appartenance sociale ou culturelle ou des conditions matérielles de vie qu'elles ont connues par le passé et dans la suite de leur parcours : "Fallait élever les enfants dans un climat très... très zen. En fin de compte mon fils il peut y avoir quarante personnes ici, il dort hein! Il est habitué au bruit. Mais ça, eux non. Moi je voulais habituer mes enfants au bruit, pour eux faut pas, faut pas faire ça. Bon plein de petites choses comme ça mais à force... Fallait faire des massages aux enfants, prendre le contact avec eux... Ça on avait le droit à des cours, on a eu des cours obligatoires. Moi je m'en fous, j'aimais bien, fallait mettre le bébé sur une table pour le masser avec de l'huile d'amande douce et tout... Moi j'aimais bien, mais c'était obligatoire. C'était pas adapté».

Le regard des professionnel-le-s sur les relations entre la mère et son enfant et sur le développement de celui-ci occupe donc une grande place dans les récits des femmes. Il est très délicat pour ces femmes d'accepter un questionnement ou une intervention éducative centrée 
sur la relation aux enfants : «je suis gentille mais quand on s'en prend à mes enfants, ça c'est sacré, comme on dit, les enfants, enfin pour moi pour les autres personnes je ne sais pas, mais enfin pour moi voilà c'est sacré, voilà c'est comme ça». Pour elles, l'observation des relations mère-enfant détermine en grande partie la qualité des relations qu'elles entretiennent elles-mêmes avec les professionnelles. Si la mère est jugée « autonome » avec son enfant, elle n'a pas de problèmes avec les professionnel-le-s. Au contraire, si les professionnel-le-s abordent des difficultés dans la prise en charge de l'enfant, la plupart des femmes rencontrées réagissent fortement. D'ailleurs, pour une partie des femmes rencontrées, un enfant ne présentant pas de problèmes est le signe que la mère est « une bonne mère ». Nombreuses sont les femmes qui nous ont affirmé : "tout allait bien avec eux, ils voyaient que ça se passait bien avec l'enfant, ils ont vu que j'arrivais à me débrouiller, donc tout allait bien ».

Ces femmes mettent en avant leurs capacités maternelles pour se distancier du vécu de surveillance dans le cadre de la protection de l'enfance. En effet, sur le plan symbolique, l'admission de la mère à l'Aide sociale à l'enfance en raison de l'entrée en centre maternel suscite des représentations fortes chez nos interlocutrices. La crainte du placement de l'enfant est évoquée par environ un quart des femmes rencontrées. Pour certaines, ces représentations s'ancrent dans une expérience du placement dans leur enfance ou adolescence : «tu te dis dans ta tête attends je suis dans un centre maternel, tu te dis la DDASS tu vois... Parce que moi quand on était jeunes on était placés, on était à la DDASS. (...) Je pense c'est plus le vécu qu'on a eu là-bas qui me faisait penser que si tu fais une faute avec ton enfant ou quelque chose comme ça, tu peux te le faire retirer». Cette crainte du placement est alimentée par des cas concrets de placement d'enfant durant leur séjour, souvent dans un contexte de crise. Ces épisodes semblent générer une crainte plus importante chez les femmes qui maîtrisent le moins les logiques du dispositif, notamment les migrantes : " on arrache les enfants et puis on les donne à d'autres personnes et tout et tout, il y a une petite fille congo-zaïroise là, mais elle était vraiment vraiment abattue, elle pleurait comme un animal dans une forêt, c'est pas possible, on lui a pris son enfant, on allait l'amener jusqu'à l'Aide sociale à l'enfance, la petite elle pleurait et ils l'ont prise ».

Si toutes les femmes rencontrées accueillies après l'âge de 21 ans partagent ces réticences sur l'intervention éducative sur la relation mère-enfant, qu'elles aient vécu un parcours migratoire ou non, en revanche elles ne maîtrisent pas toutes de la même façon les logiques 
du dispositif. Cette différence est notamment perceptible dans l'expérience qu'elles font de l'injonction à parler de soi dans le cadre du suivi.

Une répétition sans signification pour les femmes les plus suivies

Du fait d'une longue fréquentation des services sociaux, certaines femmes ont une grande habitude des suivis socio-éducatifs. Elles sont en capacité de répondre à l'injonction de parler de soi, mais cette parole n'a aucun sens et n'est pas vécue comme aidante. Une femme suivie depuis son enfance par des services sociaux, explique qu'elle allait voir la psychologue du centre «pour faire plaisir » et pour boire le café, mais que cela n'avait pas d'intérêt pour elle : «depuis que j'ai l'âge de six ou sept ans, je fais que ça de raconter ma vie. Bon à chaque fois y a un paragraphe en plus, donc je rajoute le paragraphe, mais... je raconte ma vie comme une histoire, j'ai l'impression que c'est même plus la mienne, je raconte une histoire que je connais par cour. Et je la raconte, je la raconte... mais sans intérêt! On dirait que c'est une poésie que j'ai apprise par cour et que je la récite à chaque personne que je dois rencontrer». Connaissant bien les institutions sociales, elle explique se plier à cette contrainte pour éviter des difficultés : "je faisais pas pour moi-même, je faisais pas par plaisir ou par envie, mais pour faire plaisir, pour dire "regardez je l'ai fait maintenant foutez-moi la paix. Vous voulez que je vois des psychologues, vous voulez que je fasse ci, vous voulez que je fasse des sorties, bon je les ai faites, vous êtes contents, maintenant lâchez$m o i " ~ »$. Cette anticipation de difficultés possibles avec l'institution est tout à fait réaliste, comme on le verra par la suite.

\section{La difficulté des migrantes face à l'injonction biographique}

$\mathrm{Au}$ contraire, pour les femmes arrivées récemment en France après un parcours migratoire, la nécessité de parler de soi dans le cadre du suivi socio-éducatif est beaucoup moins habituelle. Elle est souvent vécue très difficilement. Cela peut être dû à une éducation construite sur d'autres normes culturelles :

«Des fois les rendez-vous se passent pas bien. C'est à cause de ça, on n'a pas forcément... on n'est pas habitué à trop parler, y a une éducation qu'on a eue et puis... Moi avant de venir j'ai pas eu l'habitude de raconter ma vie, de parler, pour moi c'est comme si on m'en demandait trop. J'ai du mal à parler parce que c'est l'éducation qu'on m'a donnée, on m'a pas appris à me... on va dire à me valoriser, c'est ça, parler. Et la plupart du temps les Africaines que ce 
soit arabes ou noires que vous allez rencontrer, c'est comme ça, on est plutôt renfermées, c'est pas parce qu'on veut pas mais c'est comme ça, on nous a pas appris à nous valoriser, à parler tranquillement avec confiance, c'est tout le temps c'est le silence quoi. Si tu parles qu'on te demande rien c'est comme ça, et donc c'est plus ça qui a été dur».

Ces femmes font l'expérience d'une « injonction biographique », qui s'inscrit dans l'évolution en profondeur des politiques d'action sociale. Celles-ci développent la responsabilisation de l'individu, sommé d'être "acteur de son projet», et tentent d'adapter les dispositifs aux situations particulières : il faut être capable de mettre son histoire, son parcours, sa situation et ses projets en mots (Astier et Duvoux, 2006). Mais apprendre à parler de soi, à mettre sa trajectoire biographique en forme fait figure d'une compétence qu'elles ne possèdent pas et qu'elles doivent acquérir, sous peine de rencontrer des difficultés avec les professionnel-le-s, comme l'illustre cet extrait dans lequel une femme relate son échange avec un éducateur :

"Il s'est dit que si moi je n'allais pas le voir souvent, je n'allais pas discuter avec lui, c'est parce que je ne voulais pas travailler avec lui. J'ai dit "non, c'est pas parce que moi je ne veux pas travailler avec vous, je vous vois chaque quinze jours, chaque quinze jours c'est la même chose que je vous dis, mais j'ai rien de particulier à vous dire, et moi ça prend sur mon temps et effectivement ça m'agace de venir m'assoir là toujours dire les mêmes choses, voilà. Je vous ai dit, si ça ne va pas je viendrai vous voir pour vous dire monsieur ça va pas, j'ai besoin d'une aide", "ah bon d'accord donc c'est que ça va bien pour vous, alors vous n'avez plus votre place ici", je dis "ah bon", il me dit "oui parce que le centre maternel c'est fait pour les mamans seules, les mamans qui ont des problèmes", j'ai dit "écoutez chacun a des problèmes mais chacun gère ses problèmes, moi je sens que je suis assez forte mentalement, y a des petits problèmes que d'autres vont pas pouvoir gérer, ils vont vous voir, mais moi y a des petits problèmes que je peux gérer, donc je viendrai vous voir quand j'aurais de gros, gros problèmes", "ah oui mais dans ce cas vous n'avez plus votre place ici si vous n'avez pas de problème».

Il existe ainsi un enjeu fort autour du maintien de l'hébergement de la femme et de son enfant au centre maternel. 


\section{L'approche de la sortie : un moment de déséquilibre du mésosystème}

Le moment de la sortie du centre maternel permet d'observer les recompositions du mésosystème dans un moment de profond bouleversement qui cristallise des dynamiques relationnelles antérieures. La centration sur les relations mère-enfant est particulièrement présente: les professionnel-le-s vont devoir s'interroger sur le risque encouru par l'enfant dans un autre contexte. Lorsque la sortie approche, les choix d'hébergement faits par la femme sont lus à l'aune de la stabilité du milieu de vie qu'elle pourra proposer à son enfant, alors que les contraintes structurelles auxquelles elle doit faire face sont très fortes. L'évaluation des compétences maternelles et du développement de l'enfant par les professionnel-le-s constitue un enjeu central.

Ainsi, une jeune femme récemment arrivée en France, dont le séjour s'est bien déroulé, décide, alors qu'elle est enceinte d'un deuxième enfant, de partir s'installer avec son fils dans une petite chambre trouvée par le père de l'enfant plutôt que de rester au centre maternel. Ce projet de sortie, non soutenu par le centre maternel en raison des conditions matérielles, impactera ses relations ultérieures avec les services sociaux de secteur: "j'ai été au centre d'action sociale, ils m'avaient dit "on peut pas vous aider", parce qu'au foyer ils ont envoyé mon rapport là-bas! Comme quoi Mme X, on était avec elle, elle était tranquille et tout, elle a préféré son mari que ses enfants! Et là-bas, ils m'ont dit "on ne peut jamais vous aider, parce que t'as préféré ton mari que ta vie avec tes enfants". » Pour elle, cette décision a pourtant été dictée également par l'intérêt de son fils : "Quand il (le papa) vient me voir, il part, le petit il pleure jusqu'à ce qu'il tombe malade, tout le temps, tout le temps, tout le temps ». Dans cette situation, c'est la décision de partir s'installer avec le père des enfants dans des conditions matérielles particulièrement précaires qui remet en question les compétences et les choix parentaux de la mère aux yeux des professionnel-le-s.

Les situations de non-renouvellement de prise en charge pour non-adhésion au suivi, qui concernent cinq situations sur les quaranteneuf enquêtées, sont particulièrement symptomatiques d'un emballement des dynamiques relationnelles du mésosystème : plus les professionnel-le-s pointent des éléments à surveiller dans le développement de l'enfant, plus les femmes s'opposent à la rencontre avec les professionnel-le-s, ce qui peut, à terme, mener à des décisions de fin de prise en charge, comme pour cette femme qui verra sa relation avec les professionnel-le-s se dégrader au fil du séjour. Dans 
un premier temps, le rapport à l'alimentation de son fils alerte les professionnel-le-s : "On m'a dit "Oui il se passe quelque chose, l'enfant n'est pas normal, il réclame jamais à manger", j'ai dit mon enfant il est normal, il réclame jamais à manger c'est vrai à la fin il a jamais réclamé à manger même quand il a faim, c'est pas son problème c'est pas un gros mangeur ». Face à cette situation, des temps de rencontre et de réunions sont organisés, contre son avis : "cette réunion, moi je trouve qu'elle n'a pas lieu d'être, je vous ai déjà rencontré, la directrice, l'éducateur et tout et tout, maintenant avec le médecin, le psychologue et tout, toute une batterie... " Elle commence à éviter les rencontres avec les professionnel-le-s, et finalement devra quitter le centre maternel car elle n'adhère plus au suivi : "Je suis sortie un an avant la date prévue, (...) en mai ils m'ont appelée pour me dire: oui l'association a décidé de ne pas renouveler mon contrat parce que y a eu des problèmes. Oui parce qu'après on m'a donné un éducateur, c'est vrai que il fallait chaque mois faire un bilan tout le temps, quand y avait des besoins, il fallait voir les psychologues...».

Au moment de la sortie, cette femme et son fils seront hébergés chez une connaissance, sans qu'un relais ou un suivi ne puisse être mis en place alors que les professionnel-le-s restent inquiets pour l'évolution de l'enfant. Plus les professionnel-le-s focalisent le suivi sur les difficultés de son fils, plus la mère semble résister au suivi, plus l'institution raccourcit le séjour, aboutissant à une sortie dans des conditions instables, sans soutien et sans relais possible, alors que l'enfant est repéré comme ayant besoin de soutien.

On retrouve des dynamiques similaires dans les deux départements enquêtés et chez tous les profils de femmes accueillies. Elles mettent à jour le paradoxe de l'aide contrainte (Hardy, 2012). Les femmes n'ayant pas d'autre choix que de "vouloir l'aide qu'on a voulu qu'elles veuillent», il devient difficile pour les professionnel-le-s de distinguer les femmes «qui ont vraiment voulu de l'aide qu'on a voulu qu'elles veuillent » de celles qui ont feint de la vouloir. Même si les femmes se plient aux demandes formelles pour conserver leur place en centre maternel, elles échouent à se présenter comme "voulant changer». Plus les professionnel-le-s les sentent résistantes à l'intervention éducative, plus les difficultés de l'enfant sont pointées et plus les positions se rigidifient de part et d'autre, conduisant paradoxalement les enfants repérés comme plus vulnérables à sortir sans soutien. 


\section{Conclusion}

Finalement, l'inscription des centres maternels dans le champ de la protection de l'enfance a des conséquences sur l'ensemble de l'écosystème. $\mathrm{Au}$ niveau macrosystémique, cette inscription leur permet un niveau d'accueil et d'encadrement souvent bien supérieur à ce qui est constaté dans les centres d'hébergement relevant du secteur de l'insertion, notamment sur le plan des locaux, de l'accueil des enfants, de la qualification et du nombre des professionnel-le-s. Néanmoins, la description des processus mésosystémiques autour de l'enfant met en évidence le vécu paradoxal du suivi socio-éducatif effectué au sein des centres maternels, perçu par les mères à la fois comme un soutien et un contrôle. L'admission de la mère à l'Aide sociale à l'enfance a un impact fort sur l'ensemble des relations mésosystémiques observées, puisqu'elle place l'enfant comme un enjeu entre l'ensemble des acteurs et fait de la femme accueillie non plus une femme sans domicile mais une mère «à risque ».

Lorsque les missions du centre maternel font l'objet d'une lecture étroite, les professionnel-le-s des centres maternels et les résidentes peuvent être piégés dans un système relationnel paradoxal. En effet, pour justifier le séjour, les professionnel-le-s sont contraint-e-s d'intervenir sur la relation mère-enfant (ou au moins de l'observer). Le maintien de l'hébergement est conditionné par ce suivi éducatif, ce qui place les professionnel-le-s en position de contrôle social et d'obligation d'intervention, et la femme en position d'aide contrainte. En conséquence, le développement et le comportement de l'enfant deviennent un enjeu dans les relations entre la femme et les intervenants : l'enfant «sans problème» atteste de la compétence maternelle et renforce positivement l'identité de celle-ci. À l'inverse, certaines mères vivent comme une attaque identitaire l'identification, par les professionnel-le-s, de difficultés parfois bénignes chez leur enfant, ce qui peut empêcher la collaboration.

Le vécu de l'intervention socio-éducative par les femmes hébergées est fortement en lien avec leur parcours antérieur. Cette expérience semble être particulièrement difficile pour les migrantes adultes, qui en raison d'une moindre maîtrise des logiques du système, sont particulièrement démunies et adoptent facilement des stratégies qui conduisent au blocage du système. Au contraire, les femmes les plus habituées aux services sociaux ne remettent pas en cause cette volonté de les aider, voire de les faire changer ; lorsque l'injonction se fait trop pesante, elles se soumettent ou aménagent une porte de sortie, mais vont plus rarement jusqu'au point de rupture qui risquerait de se retourner contre elles. Les femmes les plus jeunes semblent les moins 
en difficulté avec cette inscription dans le champ de la protection de l'enfance, leur jeunesse venant légitimer l'intervention, sans que cela ne remette en cause leurs compétences maternelles.

La norme grandissante du «parentalisme» (Sellenet, 2007) s'applique fortement à ces femmes de milieu populaire dont l'insertion professionnelle est souvent difficile et peu valorisante. Dans ce contexte, affirmer le primat de la fonction maternelle sur tous les autres rôles sociaux que la femme peut endosser est un risque important. Tous les choix de la femme pourront être lus à l'aune des choix qu'elle fait pour ses enfants. Cela rejoint la centration des discours des professionnel-le-s de la protection de l'enfance sur les caractéristiques de la relation parent-enfant, plus que sur les conditions matérielles de vie des familles, observée dans l'ensemble des recherches sur la protection de l'enfance en France (Minary, 2011 ; Potin, 2012). Dans le cadre des centres maternels, la focalisation de l'intervention sur la relation mère-enfant peut être vécue comme une forme de soutien pour les femmes que cela menace moins identitairement, mais elle est parfois également productrice d'instabilité, conduisant à des situations de tensions entre les mères et les professionnel-le-s, voire à des sorties sans accompagnement pour des enfants pourtant repérés comme nécessitant un soutien dans leur développement.

\section{Références bibliographiques}

Astier, I. et Duvoux, N. (2006). La société biographique : une injonction à vivre dignement. Paris : L'Harmattan.

Boulbès, Y. (2005). L'histoire des maisons maternelles, entre secours et redressement : l'exemple du département de l'Aude. Paris : l'Harmattan.

Bronfenbrenner, U. (1996). Le modèle Processus-Personne-ContexteTemps. Dans R. Tessier et G. Tarabulsy (dir.), Le modèle écologique dans l'étude du développement de l'enfant (11-59). Ste Foy: Presses de l'Université du Québec.

Cardi, C. (2007). Le contrôle social réservé aux femmes : entre prison, justice et travail social. Déviance et Société, 31(1), 3-23.

Cardi, C. (2008). La déviance des femmes : délinquantes et mauvaises mères. Entre prison, justice et travail social. Thèse de doctorat en sociologie. Université Paris Diderot - Paris 7.

Corbillon, M., Assailly J.-P. et Duyme M. (1990). L'enfant placé. De l'Assistance publique à l'Aide sociale à l'enfance. Paris : Ministère de la Solidarité, de la Santé et de la Protection Sociale. 
Corbillon, M. et Duléry, A. (1997). Étude des interventions d'aide en direction des mères isolées. Rapport du Centre de Recherche en Éducation et en Formation - Université Paris X.

Donati, P., Mollo-Bouvier, S., Norvez, A. et Rollet-Echalier, C. (1999). Les centres maternels : réalités et enjeux éducatifs. Paris : L'Harmattan.

Dumaret, A. C. (2003). Soins médicosociaux précoces et accompagnement psychoéducatif : revue de la littérature étrangère. Archives de Pédiatrie, 10(5), 448-461.

Durning, P. (1995). Éducation familiale; acteurs, processus et enjeux. Paris : PUF.

Foll, J. L. et Guedeney, A. (2014). Visites à domicile préventives périnatales dans les familles à problèmes multiples : efficacité et limites. Devenir, 26(1), 59-72.

Ganne, C. et Bergonnier-Dupuy, G. (2011). Le devenir des enfants accueillis avec leur mère en centre maternel au cours de leurs premières années de vie. Rapport pour l'Observatoire national de l'enfance en danger. Université Paris Ouest Nanterre la Défense.

Hardy, G. (2012). S'il te plaît, ne m'aide pas ! : l'aide sous injonction administrative ou judiciaire. Ramonville Saint-Agne : Erès.

Korfmacher, J. (2002). Le sens caché des programmes d'interventions précoces. Devenir, 14(4), 363-375.

Minary, J.-P. (2011). Contextes de vie, précarité et protection de l'enfance. Dans M. Boutanquoi (dir.), Interventions sociales auprès des familles en situation de précarité (p. 73-97). Paris : L'Harmattan.

Ministère de la Santé et de la Sécurité Sociale (1981). Politique d'aide sociale à l'enfance : circulaire No 81/5 du 23 janvier 1981.

Neyrand, G. (2011). Soutenir et contrôler les parents : le dispositif de parentalité. Toulouse : Érès.

Pliquet, E. (2015a). Plus de 100000 places d'hébergement pour les personnes en difficulté sociale. Études et résultats, 916.

Pliquet, E. (2015b). L'hébergement social, hors urgence : plus de 81000 personnes sans logement accueillies en établissements. Études et résultats, 918.

Potin, E. (2012). Enfants placés, déplacés, replacés: parcours en protection de l'enfance. Toulouse : Érès.

Sellenet, C. (2007). La parentalité décryptée : pertinence et dérives d'un concept. Paris : l'Harmattan.

Wendland, J. (2010). Le travail des centres maternels auprès de jeunes mères célibataires à haut risque. L'Évolution psychiatrique, 75(2), 249-259. 
Wilpert, M.-D. (2013). Tous les chemins professionnels mènent-ils à la mère ? Dans G. Neyrand, M.-D. Wilpert et M. Tort (dir.), Père, mère, des fonctions incertaines. Les parents changent, les normes restent? (p. 43-82). Toulouse : Erès. 